\title{
Time Frequency Method of Solving One Boundary Value Problem for a Hyperbolic System and Its Application to the Oil Extraction
}

\author{
F.A. Aliev, N.A. Aliev, and A.P. Guliev \\ Institute of Applied Mathematics, Baku State University \\ 23, Z. Khalilov Str., Baku AZ1148, Azerbaijan \\ E-mail: f_aliev@yahoo.com
}

Received May 29, 2014, revised November 30, 2015

\begin{abstract}
We consider the boundary value problem, where the motion of the object is described by the two-dimensional linear system of partial differential equations of hyperbolic type where a discontinuity is at a point within the interval that defines the phase coordinate $x$. Using the method of series and Laplace transformation in time $t$ (time-frequency method), an analytical solution is found for the determination of debit $Q(2 l, t)$ and pressure $P(2 l, t)$, which can be effective in the calculation of the coefficient of hydraulic resistance in the lift at oil extraction by gas lift method where $l$ is the well depth. For the case where the boundary functions are of exponential form, the formulas for $P(2 l, t)$ and $Q(2 l, t)$ depending only on $t$ are obtained. It is shown that at constant boundary functions, these formulas allow us to determine the coefficient of hydraulic resistance in the lift of gas lift wells, which determines the change in the dynamics of pollution.
\end{abstract}

Key words: hyperbolic equation, boundary problems, method of series, Laplace transformation, time-frequency method, gas lift, coefficient of hydraulic resistance.

Mathematics Subject Classification 2010: 65M38, 35L02, 35L40, 58J45, 58J90.

\section{Introduction}

As known [1-4], only a considerably small part of oil is extracted from the reservoir by gushing method. The rest of oil is extracted by secondary and tertiary methods (gas lifting [2-4], subsurface pumping [1, 2], etc.). As considerable volumes of oil are produced from wells by gas lift method [2, 3, 5-12], researchers have paid a lot of attention to creating mathematical models of this process 
lately. However, in [5], the authors, averaging the motion equation (in partial derivatives) with respect to time $t$ and depth $E$, solved the general problem of control and optimization for the corresponding ordinary differential equations. These simplifications make it possible to obtain "approximate" mathematical models instead of exact ones. Therefore the authors of [13] applying the difference schemes [14] and the method of series by the well depth [15] for the motion equation of gas-liquids mixture in the lift obtained the total number of terms of the series which defines the solution representation to a given accuracy. In the present paper, under some restrictions imposed on the boundary conditions [1618], an analytical form of the solution of the motion equation in the considered problem is given. In some partial cases, such a representation (when the volume of the gas supplied 'shoe' is constant) allows us to define the analytical expression of the coefficient of hydraulic resistance in the pump-compressor pipe and the change of pollution dynamics $[19,20]$.

\section{The Problem Statement and the Method of Series for Solving a Boundary Value Problem in an Annular Space}

Let us consider the boundary value problem for the system of equations of hyperbolic type

$$
\left\{\begin{array}{l}
-F_{1} \frac{\partial P_{1}(x, t)}{\partial x}=\frac{\partial Q_{1}(x, t)}{\partial t}+2 a_{1} Q_{1}(x, t), \\
-F_{1} \frac{\partial P_{1}(x, t)}{\partial t}=c_{1} \frac{\partial Q_{1}(x, t)}{\partial x},
\end{array} \quad x>0, \quad t \in R,\right.
$$

with the boundary conditions

$$
\left\{\begin{array}{l}
P_{1}(0, t)=P_{10}(t), \\
Q_{1}(0, t)=Q_{10}(t),
\end{array} \quad t \in R,\right.
$$

where $P_{1}(x, t)$ is a pressure, $Q_{1}(x, t)$ is a gas volume,

$$
2 a_{1}=\frac{g}{\omega_{c_{1}}}+\frac{\lambda \omega_{c_{1}}}{2 D_{1}}
$$

$\omega_{c_{1}}$ is an average velocity of a flow with respect to the length of the annular pipeline, $\lambda$ is the coefficient of hydraulic resistances, $D_{1}$ is the effective diameter of the annular space, $F_{1}$ is the area of the cross-section of pump-compressor pipes, which is constant along the axis $x, c_{1}$ is the sound speed in gas, $g$ is the gravity acceleration, $P_{10}(t)$ and $Q_{10}(t)$ are given continuous functions specifying the initial pressure and the volume of the gas supplied to the "shoe". Thus, it is required to find $P(x, t)$ and $Q(x, t)$ which satisfy conditions (1), (2), where $P(x, t)$ is the pressure and $Q(x, t)$ is the gas volume at each point $(x, t)$ in the pipe. 
We will search the solution of problem (1)-(3) in the form of [15, 21-23] as following from [24],

$$
\left\{\begin{array}{l}
P_{1}(x, t)=\sum_{k=0}^{\infty} P_{1 k}(t) \frac{x^{k}}{k !}, \\
Q_{1}(x, t)=\sum_{k=0}^{\infty} Q_{1 k}(t) \frac{x^{k}}{k !}, \quad x>0, \quad t \in R,
\end{array}\right.
$$

which satisfies the boundary condition (2). Differentiating (4) and substituting it into (1), for the unknown functions $P_{1 k}(t)$ and $Q_{1 k}(t)$, for $k>0$, we have:

$$
\left\{\begin{array}{l}
P_{1,2 k}(t)=\left(\frac{d}{d t}+2 a_{1}\right)^{k} \frac{P_{10}^{(k)}(t)}{c_{1}^{k}}, \\
Q_{1,2 k}(t)=\left(\frac{d}{d t}+2 a_{1}\right)^{k} \frac{Q_{10}^{(k)}(t)}{c_{1}^{k}}, \quad k \geq 0,
\end{array}\right.
$$

and

$$
\left\{\begin{array}{l}
P_{1,2 k+1}(t)=-\left(\frac{d}{d t}+2 a_{1}\right)^{k+1} \frac{Q_{10}^{(k)}(t)}{F_{1} c_{1}^{k}}, \\
Q_{1,2 k+1}(t)=-\left(\frac{d}{d t}+2 a_{1}\right)^{k} \frac{F_{1} P_{10}^{(k+1)}(t)}{c_{1}^{k+1}}, \quad k \geq 0 .
\end{array}\right.
$$

Introduce the Laplace transforms [25]:

$$
\left\{\begin{array}{l}
\tilde{P}_{10}(\rho)=\int_{0}^{\infty} e^{-\rho t} P_{10}(t) d t \\
\tilde{Q}_{10}(\rho)=\int_{0}^{\infty} e^{-\rho t} Q_{10}(t) d t
\end{array}\right.
$$

allowing one to recover $P_{10}$ and $Q_{10}$ by the the formulas

$$
\left\{\begin{array}{l}
P_{10}(t)=\frac{1}{2 \pi i} \int_{L_{1}} e^{\rho t} \tilde{P}_{10}(\rho) d \rho \\
Q_{10}(t)=\frac{1}{2 \pi i} \int_{L_{1}} e^{\rho t} \tilde{Q}_{10}(\rho) d \rho,
\end{array}\right.
$$

where $L_{1}=\rho: \operatorname{Re} \rho=\sigma_{1}>0$. Then, taking into account (8), in (5) and (6) we obtain

$$
\left\{\begin{array}{l}
P_{1,2 k}(t)=\frac{1}{2 \pi i} \int_{L_{1}} e^{\rho t}\left(\rho+2 a_{1}\right)^{k} \rho^{k} \tilde{P}_{10}(\rho) \frac{d \rho}{c_{1}^{k}} \\
Q_{1,2 k}(t)=\frac{1}{2 \pi i} \int_{L_{1}} e^{\rho t}\left(\rho+2 a_{1}\right)^{k} \rho^{k} \tilde{Q}_{10}(\rho) \frac{d \rho}{c_{1}^{k}}
\end{array}\right.
$$

and

$$
\left\{\begin{array}{l}
P_{1,2 k+1}(t)=-\frac{1}{2 \pi i} \int_{L_{1}} e^{\rho t}\left(\rho+2 a_{1}\right)^{k+1} \rho^{k} \tilde{Q}_{10}(\rho) \frac{d \rho}{F_{1} c_{1}^{k}}, \\
Q_{1,2 k+1}(t)=-\frac{1}{2 \pi i} \int_{L_{1}} e^{\rho t}\left(\rho+2 a_{1}\right)^{k} F_{1} \rho^{k+1} \tilde{P}_{10}(\rho) \frac{d \rho}{c_{1}^{k+1}} .
\end{array}\right.
$$

Thus, in (9), (10) and (4), we have the following analytic expressions for the solution of equation (1):

$$
\begin{aligned}
& P_{1}(x, t)=\sum_{k=0}^{\infty} P_{1,2 k}(t) \frac{x^{2 k}}{(2 k) !}+\sum_{k=0}^{\infty} P_{1,2 k+1}(t) \frac{x^{2 k+1}}{(2 k+1) !} \\
& =\frac{1}{2 \pi i} \int_{L_{1}} e^{\rho t}\left\{\tilde{P}_{10}(\rho) \operatorname{ch}\left(\sqrt{\frac{\rho\left(\rho+2 a_{1}\right)}{c_{1}}} x\right)-\frac{\tilde{Q}_{10}(\rho)}{F_{1}} \sqrt{\frac{c_{1}\left(\rho+2 a_{1}\right)}{\rho}} \operatorname{sh}\left(\sqrt{\frac{\rho\left(\rho+2 a_{1}\right)}{c_{1}}} x\right)\right\} d \rho
\end{aligned}
$$




$$
\begin{aligned}
& Q_{1}(x, t)=\sum_{k=0}^{\infty} Q_{1,2 k}(t) \frac{x^{2 k}}{(2 k) !}+\sum_{k=0}^{\infty} Q_{1,2 k+1}(t) \frac{x^{2 k+1}}{(2 k+1) !} \\
& =\frac{1}{2 \pi i} \int_{L_{1}} e^{\rho t}\left\{\tilde{Q}_{10}(\rho) \operatorname{ch}\left(\sqrt{\frac{\rho\left(\rho+2 a_{1}\right)}{c_{1}}} x\right)-\tilde{P}_{10}(\rho) F_{1} \sqrt{\frac{\rho}{c_{1}\left(\rho+2 a_{1}\right)}} \operatorname{sh}\left(\sqrt{\frac{\rho\left(\rho+2 a_{1}\right)}{c_{1}}} x\right)\right\} d \rho
\end{aligned}
$$

which can be formulated as the theorem below.

Theorem 1. If $F_{1}, a_{1}, c_{1}$ are constant, $P_{10}(t)$ and $Q_{10}(t)$ are infinitely differentiable functions, then BVP (1)-(2) has a solution analytically represented in (11) and (12). The proof of Theorem 1 follows from (11) and (12). form:

From (11) and (12) at $x=l$, we define $P_{1}(l-0, t)$ and $Q_{1}(l-0, t)$ in the

$$
\left\{\begin{array}{l}
P_{1}(l-0, t)=P_{1}(l, t)=\frac{1}{2 \pi i} \int e^{\rho t}\left\{\tilde{P}_{10}(\rho) \operatorname{ch}(\mu l)-\frac{\tilde{Q}_{10}(\rho)}{F_{1}} \sqrt{\frac{c_{1}\left(\rho+2 a_{1}\right)}{\rho}} \operatorname{sh}(\mu l)\right\} d \rho \\
Q_{1}(l-0, t)=Q_{1}(l, t)=\frac{1}{2 \pi i} \int_{L_{1}} e^{\rho t}\left\{\tilde{Q}_{10}(\rho) \operatorname{ch}(\mu l)-\tilde{P}_{10}(\rho) F_{1} \sqrt{\frac{\rho}{c_{1}\left(\rho+2 a_{1}\right)}} \operatorname{sh}(\mu l)\right\} d \rho
\end{array}\right.
$$

where $(\mu)=\left(\sqrt{\frac{\rho\left(\rho+2 a_{1}\right)}{c_{1}}} l\right)$.

\section{The Solution of Equation (1) in the Lift $x>l$}

Now we pass from the end of the first stage to the initial position of the second stage, i.e., let the hyperbolic equation $(1)$ at $(l, t)$ have discontinuity [26],

$$
\left\{\begin{array}{l}
P_{2}(l+0, t)=P_{2}(l, t)=F_{\delta}^{1} P_{1}(l-0, t)+\chi_{1}\left(P_{1}(l-0, t), \alpha_{1}, \alpha_{2}, \alpha_{3}\right) \bar{P}(t) \equiv P_{20}(t), \\
Q_{2}(l+0, t)=Q_{2}(l, t)=F_{\delta}^{2} Q_{1}(l-0, t)+\chi_{2}\left(Q_{1}(l-0, t), \beta_{1}, \beta_{2}, \beta_{3}\right) \bar{Q}(t) \equiv Q_{20}(t),
\end{array}\right.
$$

where $\chi_{1}(\cdot), \chi_{2}(\cdot)$ are continuous functions of their arguments, $\bar{P}(t), \bar{Q}(t)$ are given continuous functions that are a possible balance of oil products [9], $F_{\delta}^{i}(i=$ $1,2), \alpha_{k}, \beta_{k}(k=\overline{1,3})$ are real numbers to be determined by using the corresponding identification problems for creation of gas-liquid mixture in the well bottom zone.

Now we pass to the second stage. Let us consider the boundary value problem (1)-(3) for a system of equations of hyperbolic type

$$
\left\{\begin{array}{l}
-F_{2} \frac{\partial P_{2}(x, t)}{\partial x}=\frac{\partial Q_{2}(x, t)}{\partial t}+2 a_{2} Q_{2}(x, t), \\
-F_{2} \frac{\partial P_{2}(x, t)}{\partial t}=c_{2} \frac{\partial Q_{2}(x, t)}{\partial x}, \quad x>l, t \in R,
\end{array}\right.
$$

with the boundary conditions

$$
\left\{\begin{array}{l}
P_{2}(l, t)=P_{20}(t), \\
Q_{2}(l, t)=Q_{20}(t), \quad t \in R,
\end{array}\right.
$$


where $P_{20}(t)$ and $Q_{20}(t)$ are given in the form of (14). Then system (15) and unknowns $P_{2}(\cdot), Q_{2}(\cdot)$ are similar to those of the previous case, and $a_{2}$, similarly to $k$ in (3), will be

$$
2 a_{2}=\frac{g}{\omega_{2}}+\frac{\lambda \omega_{2}}{2 D_{2}}
$$

Analogously to the previous case from Sec. 2, the solution of the boundary problem (15)-(17) will be sought in the form of [15]:

$$
\left\{\begin{array}{l}
P_{2}(x, t)=\sum_{k=0}^{\infty} P_{2 k}(t) \frac{x^{k}}{k !}, \\
Q_{2}(x, t)=\sum_{k=0}^{\infty} Q_{2 k}(t) \frac{x^{k}}{k !}, \quad x>l, t \in R,
\end{array}\right.
$$

for which the boundary conditions (16) are satisfied automatically, and $P_{2 k}(t)$ and $Q_{2 k}(t)$ are to be defined for $k>0$. If not to repeat the calculations given above for solving (18), we can get the following expressions:

$$
\left\{\begin{array}{l}
P_{2}(x, t)=\frac{1}{2 \pi i} \int_{L_{2}} e^{r t}\left\{\tilde{P}_{20}(r) \operatorname{ch}(\mu x)-\frac{\tilde{Q}_{20}(r)}{F_{2}} \sqrt{\frac{c_{2}\left(r+2 a_{2}\right)}{r}} \operatorname{sh}(\mu x)\right\} d r \\
Q_{2}(x, t)=\frac{1}{2 \pi i} \int_{L_{2}} e^{r t}\left\{\tilde{Q}_{20}(r) \operatorname{ch}(\mu x)-\tilde{P}_{20}(r) F_{2} \sqrt{\frac{r}{c_{2}\left(r+2 a_{2}\right)}} \operatorname{sh}(\mu x)\right\} d r
\end{array}\right.
$$

where $\mu=\left(\sqrt{\frac{r\left(r+2 a_{2}\right)}{c_{2}}} x,\right)$, which, for $x=2 l$, have the form

$$
\left\{\begin{array}{l}
P_{2}(2 l, t)=\frac{1}{2 \pi i} \int_{L_{2}} e^{r t}\left\{\tilde{P}_{20}(r) \operatorname{ch}(\mu 2 l)-\frac{\tilde{Q}_{20}(r)}{F_{2}} \sqrt{\frac{c_{2}\left(r+2 a_{2}\right)}{r}} \operatorname{sh}(\mu 2 l)\right\} d r \\
Q_{2}(2 l, t)=\frac{1}{2 \pi i} \int_{L_{2}} e^{r t}\left\{\tilde{Q}_{20}(r) \operatorname{ch}(\mu 2 l)-\tilde{P}_{20}(r) F_{2}\left(\sqrt{\frac{r}{c_{2}\left(r+2 a_{2}\right)}}\right) \operatorname{sh}(\mu 2 l)\right\} d r
\end{array}\right.
$$

where $\mu=\left(\sqrt{\frac{r\left(r+2 a_{2}\right)}{c_{2}}} 2 l\right), L_{2}$, like $L_{1}$, is a Laplace line, i.e., if $r \in L_{2}$, then $\operatorname{Rer}=\sigma_{2}>0$. Thus, the following theorem is proved:

Theorem 2. Under the conditions of Theorem 1, if $F_{2}, a_{2}, c_{2}$ are constant numbers, $P_{20}(t)$ and $Q_{20}(t)$ are infinitely differentiable functions, then $B V P$ (15)-(16) has the solutions represented in the analytical form (19).

\section{Simplified Scheme}

It should be noted that if the boundary functions $P_{0}(t), Q_{0}(t)$ have an exponential form, then the solutions (11), (12) and (19) become much more simplified. In fact, let

$$
\left\{\begin{array}{l}
P_{10}(t)=\alpha_{1} e^{\nu_{1} t} \\
Q_{10}(t)=\beta_{1} e^{\mu_{1} t}, \quad t \in R .
\end{array}\right.
$$


Then

$$
\tilde{P}_{10}(\rho)=\int_{0}^{\infty} e^{-\rho t} P_{10}(t) d t=\int_{0}^{\infty} \alpha_{1} e^{\nu_{1} t} e^{-\rho t} d t=\alpha_{1} \int_{0}^{\infty} e^{-\left(\rho-\nu_{1}\right) t} d t
$$

where the constants $\alpha_{1}, \beta_{1}, \nu_{1}, \mu_{1}$ are given real numbers. If $\operatorname{Re} \rho=\sigma_{1}>\nu_{1}$, then

$$
\left\{\begin{array}{l}
\tilde{P}_{10}(\rho)=\left.\alpha_{1} \frac{e^{-\left(\rho-\nu_{1}\right) t}}{-\left(\rho-\nu_{1}\right)}\right|_{t=0} ^{\infty}=\frac{\alpha_{1}}{\rho-\nu_{1}} \\
\tilde{Q}_{10}(\rho)=\frac{\beta_{1}}{\rho-\mu_{1}} .
\end{array}\right.
$$

Substituting (22) into (11), (12) and taking into account the analyticity of the functions $\operatorname{ch}\left(\sqrt{\frac{\rho\left(\rho+2 a_{1}\right)}{c_{1}}} x\right)$ and $\sqrt{\frac{c_{1}\left(\rho+2 a_{1}\right)}{\rho}} \operatorname{sh}\left(\sqrt{\frac{\rho\left(\rho+2 a_{1}\right)}{c_{1}}} x\right)$, via the variable $\rho$ we find

$$
\left\{\begin{array}{l}
P_{1}(x, t)=\alpha_{1} e^{\nu_{1} t} \operatorname{ch}\left(\sqrt{\frac{\nu_{1}\left(\nu_{1}+2 a_{1}\right)}{c_{1}}} x\right)-\beta_{1} e^{\mu_{1} t} \frac{1}{F_{1}} \sqrt{\frac{c_{1}\left(\mu_{1}+2 a_{1}\right)}{\mu_{1}}} \operatorname{sh}\left(\sqrt{\frac{\mu_{1}\left(\mu_{1}+2 a_{1}\right)}{c_{1}}} x\right), \\
Q_{1}(x, t)=\beta_{1} e^{\mu_{1} t} \operatorname{ch}\left(\sqrt{\frac{\mu_{1}\left(\mu_{1}+2 a_{1}\right)}{c_{1}}} x\right)-\alpha_{1} e^{\nu_{1} t} F_{1} \sqrt{\frac{\nu_{1}}{c_{1}\left(\nu_{1}+2 a_{1}\right)}} \operatorname{sh}\left(\sqrt{\frac{\nu_{1}\left(\nu_{1}+2 a_{1}\right)}{c_{1}}} x\right) .
\end{array}\right.
$$

Thus, expression (13) finally takes the form

$$
\left\{\begin{array}{l}
P_{1}(l, t)=\alpha_{1} e^{\nu_{1} t} \operatorname{ch}\left(\sqrt{\frac{\nu_{1}\left(\nu_{1}+2 a_{1}\right)}{c_{1}}} l\right)-\beta_{1} e^{\mu_{1} t} \frac{1}{F_{1}} \sqrt{\frac{c_{1}\left(\mu_{1}+2 a_{1}\right)}{\mu_{1}}} \operatorname{sh}\left(\sqrt{\frac{\mu_{1}\left(\mu_{1}+2 a_{1}\right)}{c_{1}}} l\right) \\
Q_{1}(l, t)=\beta_{1} e^{\mu_{1} t} \operatorname{ch}\left(\sqrt{\frac{\mu_{1}\left(\mu_{1}+2 a_{1}\right)}{c_{1}}} l\right)-\alpha_{1} e^{\nu_{1} t} F_{1} \sqrt{\frac{c_{1}\left(\nu_{1}+2 a_{1}\right)}{\nu_{1}}} \operatorname{sh}\left(\sqrt{\frac{\nu_{1}\left(\nu_{1}+2 a_{1}\right)}{c_{1}}} l\right) .
\end{array}\right.
$$

Now we suggest that from (14) we have

$$
\left\{\begin{array}{l}
P_{20}(t)=P_{1}^{2}(l, t) \cdot P_{2}, \\
Q_{20}(t)=-Q_{1}^{2}(l, t) Q_{2},
\end{array}\right.
$$

where $P_{2}>0$ and $Q_{2}>0$ are constant numbers. Then

$$
\begin{aligned}
& \tilde{P}_{20}(r)=\int_{0}^{\infty} e^{-r t} P_{20}(t) d t=P_{2} \alpha_{1}^{2} \operatorname{ch}^{2}\left(\sqrt{\frac{\nu_{1}\left(\nu_{1}+2 a_{1}\right)}{c_{1}}} l\right) \frac{1}{r-2 \nu_{1}} \\
& -2 \alpha_{1} \beta_{1} P_{2} \frac{1}{F_{1}} \sqrt{\frac{c_{1}\left(\mu_{1}+2 a_{1}\right)}{\mu_{1}}} \operatorname{sh}\left(\sqrt{\frac{\mu_{1}\left(\mu_{1}+2 a_{1}\right)}{c_{1}}} l\right) \operatorname{ch}\left(\sqrt{\frac{\nu_{1}\left(\nu_{1}+2 a_{1}\right)}{c_{1}}} l\right) \\
& \times \frac{1}{r-\left(\nu_{1}+\mu_{1}\right)}+P_{2} \beta_{1}^{2} \frac{1}{F_{1}^{2}} \frac{c_{1}\left(\mu_{1}+2 a_{1}\right)}{\mu_{1}} \operatorname{sh}^{2}\left(\sqrt{\frac{\mu_{1}\left(\mu_{1}+2 a_{1}\right)}{c_{1}}} l\right) \frac{1}{r-2 \mu_{1}},
\end{aligned}
$$




$$
\begin{aligned}
& \tilde{Q}_{20}(r)=\int_{0}^{\infty} e^{-r t} Q_{20}(t) d t=-Q_{2} \beta_{1}^{2} \operatorname{ch}^{2}\left(\sqrt{\frac{\mu_{1}\left(\mu_{1}+2 a_{1}\right)}{c_{1}}} l\right) \frac{1}{r-2 \mu_{1}} \\
& +2 \alpha_{1} \beta_{1} Q_{2} F_{1} \sqrt{\frac{\nu_{1}}{c_{1}\left(\nu_{1}+2 a_{1}\right)}} \operatorname{sh}\left(\sqrt{\frac{\nu_{1}\left(\nu_{1}+2 a_{1}\right)}{c_{1}}} l\right) \operatorname{ch}\left(\sqrt{\frac{\mu_{1}\left(\mu_{1}+2 a_{1}\right)}{c_{1}}} l\right) \frac{1}{r-\left(\nu_{1}+\mu_{1}\right)} \\
& -Q_{2} \alpha_{1}^{2} F_{1}^{2} \frac{\nu_{1}}{c_{1}\left(\mu_{1}+2 a_{1}\right)} \operatorname{sh}^{2}\left(\sqrt{\frac{\nu_{1}\left(\nu_{1}+2 a_{1}\right)}{c_{1}}} l\right) \frac{1}{r-2 \nu_{1}} .
\end{aligned}
$$

Here it is supposed that $\operatorname{Re} r>\max \left\{2 \nu_{1},\left(\nu_{1}+\mu_{1}\right), 2 \mu_{1}\right\}$. At last, from (20), we obtain

$$
\begin{aligned}
& P_{2}(2 l, t)=P_{2} \alpha_{1}^{2} \operatorname{ch}\left(\sqrt{\frac{2 \nu_{1} 2\left(\nu_{1}+a_{2}\right)}{c_{2}}} 2 l\right) \operatorname{ch}^{2}\left(\sqrt{\frac{\nu_{1}\left(\nu_{1}+2 a_{1}\right)}{c_{1}}} l\right) e^{2 \nu_{1} t} \\
& -2 \alpha_{1} \beta_{1} P_{2} \frac{1}{F_{1}} \sqrt{\frac{c_{1}\left(\mu_{1}+2 a_{1}\right)}{\mu_{1}}} \operatorname{sh}\left(\sqrt{\frac{\mu_{1}\left(\mu_{1}+2 a_{1}\right)}{c_{1}}} l\right) \operatorname{ch}\left(\sqrt{\frac{\nu_{1}\left(\nu_{1}+2 a_{1}\right)}{c_{1}}} l\right) \\
& \times \operatorname{ch}\left(\sqrt{\frac{\left(\nu_{1}+\mu_{1}\right)\left(\nu_{1}+\mu_{1}+2 a_{1}\right)}{c_{2}}} 2 l\right) e^{\left(\nu_{1}+\mu_{1}\right) t}+P_{2} \beta_{1}^{2} \frac{1}{F_{1}^{2}} \frac{c_{1}\left(\mu_{1}+2 a_{1}\right)}{\mu_{1}} \\
& \times \operatorname{sh}^{2}\left(\sqrt{\frac{\mu_{1}\left(\mu_{1}+2 a_{1}\right)}{c_{1}}} l\right) \operatorname{ch}\left(\sqrt{\frac{2 \mu_{1} 2\left(\mu_{1}+a_{2}\right)}{c_{2}}} 2 l\right) e^{2 \mu_{1} t}+Q_{2} \beta_{1}^{2} \frac{1}{F_{2}} \sqrt{\frac{c_{2}\left(\mu_{1}+a_{2}\right)}{\mu_{1}}} \\
& \times \operatorname{sh}\left(\sqrt{\frac{2 \mu_{1} 2\left(\mu_{1}+a_{2}\right)}{c_{2}}} l\right) \operatorname{ch}^{2}\left(\sqrt{\frac{\mu_{1}\left(\mu_{1}+2 a_{1}\right)}{c_{1}}} l\right) e^{2 \mu_{1} t}-2 \alpha_{1} \beta_{1} Q_{2} \frac{F_{1}}{F_{2}} \\
& \times \sqrt{\frac{\nu_{1}}{c_{1}\left(\nu_{1}+2 a_{1}\right)}} \operatorname{sh}\left(\sqrt{\frac{\nu_{1}\left(\nu_{1}+2 a_{1}\right)}{c_{1}}} l\right) \operatorname{ch}\left(\sqrt{\frac{\mu_{1}\left(\mu_{1}+2 a_{1}\right)}{c_{1}}} l\right) \sqrt{\frac{c_{2}\left(\nu_{1}+\mu_{1}+2 a_{2}\right)}{\nu_{1}+\mu_{1}}} \\
& \times \operatorname{sh}\left(\sqrt{\frac{\left(\nu_{1}+\mu_{1}\right)\left(\nu_{1}+\mu_{1}+2 a_{2}\right)}{c_{2}} 2 l}\right) e^{\left(\nu_{1}+\mu_{1}\right) t}+Q_{2} \alpha_{1}^{2} F_{1}^{2} \frac{\nu_{1}}{c_{1}\left(\nu_{1}+2 a_{1}\right)} \\
& \times \operatorname{sh}^{2}\left(\sqrt{\frac{\nu_{1}\left(\nu_{1}+2 a_{1}\right)}{c_{1}}} l\right) \frac{1}{F_{2}} \sqrt{\frac{c_{2}\left(\nu_{1}+a_{2}\right)}{\nu_{1}}} \operatorname{sh}\left(\sqrt{\frac{2 \nu_{1} 2\left(\nu_{1}+a_{2}\right)}{c_{2}}} 2 l\right) e^{2 \nu_{1} t}, \\
& Q_{2}(2 l, t)=-Q_{2} \beta_{1}^{2} \operatorname{ch}^{2}\left(\sqrt{\frac{\mu_{1}\left(\mu_{1}+2 a_{1}\right)}{c_{1}}} l\right) \operatorname{ch}\left(\sqrt{\frac{2 \mu_{1} 2\left(\mu_{1}+a_{2}\right)}{c_{2}}} l l\right) e^{2 \mu_{1} t} \\
& +2 \alpha_{1} \beta_{1} Q_{2} F_{1} \sqrt{\frac{\nu_{1}}{c_{1}\left(\nu_{1}+2 a_{1}\right)}} \operatorname{sh}\left(\sqrt{\frac{\nu_{1}\left(\nu_{1}+2 a_{1}\right)}{c_{1}}} l\right) \operatorname{ch}\left(\sqrt{\frac{\mu_{1}\left(\mu_{1}+2 a_{1}\right)}{c_{1}}} l\right) \\
& \times \operatorname{ch}\left(\sqrt{\frac{\left(\nu_{1}+\mu_{1}\right)\left(\nu_{1}+\mu_{1}+2 a_{2}\right)}{c_{2}}} 2 l\right) e^{\left(\nu_{1}+\mu_{1}\right) t}-Q_{2} \alpha_{1}^{2} F_{1}^{2} \frac{\nu_{1}}{c_{1}\left(\nu_{1}+2 a_{1}\right)} \\
& \times \operatorname{sh}^{2}\left(\sqrt{\frac{\nu_{1}\left(\nu_{1}+2 a_{1}\right)}{c_{1}}} l\right) \operatorname{ch}\left(\sqrt{\frac{2 \nu_{1} 2\left(\nu_{1}+a_{2}\right)}{c_{2}}} 2 l\right) e^{2 \nu_{1} t}-P_{2} \alpha_{1}^{2} \\
& \times \operatorname{ch}^{2}\left(\sqrt{\frac{\nu_{1}\left(\nu_{1}+2 a_{1}\right)}{c_{1}}} l\right) F_{2}\left(\sqrt{\frac{\nu_{1}}{c_{2}\left(\nu_{1}+a_{2}\right)}}\right) \operatorname{sh}\left(\sqrt{\frac{2 \nu_{1} 2\left(\nu_{1}+a_{2}\right)}{c_{2}}} l l\right) e^{2 \nu_{1} t} \\
& +2 \alpha_{1} \beta_{1} P_{2} \frac{1}{F_{1}} \sqrt{\frac{c_{1}\left(\mu_{1}+2 a_{1}\right)}{\mu_{1}}} \operatorname{sh}\left(\sqrt{\frac{\mu_{1}\left(\mu_{1}+2 a_{1}\right)}{c_{1}} l} l\right) \operatorname{ch}\left(\sqrt{\frac{\nu_{1}\left(\nu_{1}+2 a_{1}\right)}{c_{1}}} l\right) \\
& \times F_{2}\left(\sqrt{\frac{\nu_{1}+\mu_{1}}{c_{2}\left(\nu_{1}+\mu_{1}+2 a_{2}\right)}}\right) \operatorname{sh}\left(\sqrt{\frac{\left(\nu_{1}+\mu_{1}\right)\left(\nu_{1}+\mu_{1}+2 a_{2}\right)}{c_{2}}} 2 l\right) e^{\left(\nu_{1}+\mu_{1}\right) t}
\end{aligned}
$$




$$
\begin{aligned}
& -P_{2} \beta_{1}^{2} \frac{1}{F_{1}^{2}} \frac{c_{1}\left(\mu_{1}+2 a_{1}\right)}{\mu_{1}} \operatorname{sh}^{2}\left(\sqrt{\frac{\mu_{1}\left(\mu_{1}+2 a_{1}\right)}{c_{1}}} l\right) F_{2}\left(\sqrt{\frac{\mu_{1}}{c_{2}\left(\mu_{1}+a_{2}\right)}}\right) \\
& \times \operatorname{sh}\left(\sqrt{\frac{2 \mu_{1} 2\left(\mu_{1}+a_{2}\right)}{c_{2}}} l l\right) e^{2 \mu_{1} t},
\end{aligned}
$$

which proves the theorem below.

Theorem 3. If $F_{i}, a_{i}, c_{i}, i=1,2$, are constant numbers, $P_{10}(t)$ and $Q_{10}(t)$ have the form of (21), $P_{20}(t)$ and $Q_{20}(t)$ have the form of (25), $\alpha_{1}, \beta_{1}, \nu_{1}$, $\mu_{1}, P_{2}, Q_{2}$ are constant numbers, then (28) and (29) hold true for solving $B V P$ (1) $-(3),(14)-(17)$.

\section{Determination of Coefficient of Hydraulic Resistance [19, 20]}

Now we use the relationships (28) and (29) to define $\lambda$, the coefficient of hydraulic resistance $[19,20]$ (CHR), from (16), i.e., to define the pollution dynamics in the lift. Note that this problem is essentially the inverse problem to that considered for parabolic systems in [27].

Let statistical data for the volume of the supplied gas $\tilde{Q}_{10}(t)$ from $(2)$ and debit $\tilde{Q}(2 l, t)$ be given. Then writing the functional in the form

$$
R_{Q}(\lambda)=\int_{0}^{T}[Q(2 l, t)-\tilde{Q}(2 l, t)]^{2} d t,
$$

we have to find $\lambda^{*}$ such that the functional $R(\lambda)$ gets its minimum. Therefore, we calculate the first variation of the functional (30) and make it equal zero

$$
\int_{0}^{T}[Q(2 l, t)-\tilde{Q}(2 l, t)] Q_{\lambda}^{\prime}(2 l, t) d t=0 .
$$

Taking into account (29) and (31), for defining the CHR $\lambda$ we can obtain a transcendent equation which allows us to find a numerical meaning of the optimal $\lambda^{*}$.

E x a m p l e. Let us consider BVP (1), (2) (see, [8]), where the boundary data $P_{10}(t)=P_{0}, \quad Q_{10}(t)=Q_{0}$ from (2) are constant. While in [15], in the domain $[0, l] \times[0, T]$,

$$
P(x, t)=P_{0}-2 \frac{a_{1}}{F_{1}} Q_{0} x, \quad Q(x, t)=Q_{0}
$$

and at the point $(l-0, t)$,

$$
P(l-0, t)=P_{0}-2 \frac{a_{1}}{F_{1}} Q_{0} l, \quad Q(l-0, t)=Q_{0} .
$$


Suggesting that in (14)

$$
\begin{aligned}
& \bar{P}(t)=\bar{P}, \quad \bar{Q}(t)=\bar{Q}, \quad \alpha_{1}=-1, \quad \alpha_{2}=\alpha_{3}=0, \quad \beta_{1}=1, \quad \beta_{2}=\beta_{3}=0, \\
& F_{\delta}^{1}=F_{\delta}^{2}=1, \quad \chi_{1}=P^{2}(l-0, t), \quad \chi_{2}=-Q^{2}(l-0, t),
\end{aligned}
$$

at the point $(l+0, t)$ we have (boundary layer [8])

$$
\begin{aligned}
& P(l+0, t)=P(l-0, t)+P^{2}(l-0, t) \bar{P}=P_{l+0}=\text { const } \\
& Q(l+0, t)=Q(l-0, t)-Q^{2}(l-0, t) \bar{Q}=Q_{l+0}=\text { const. }
\end{aligned}
$$

Similarly to the above, it is easy to show that in the domain $[l, 2 l] \times[0, T]$,

$$
P(x, t)=P_{l+0}-\frac{2 a_{2}}{F_{2}} Q_{l+0} x, \quad Q(x, t)=Q_{l+0} .
$$

According to (34), $Q(x, t)$ is constant on $[l, 2 l] \times[0, T]$. This makes it difficult to find the CHR $\lambda$. Besides, the expression for $P(x, t)$ contains $a_{2}(\lambda)$. This requires the necessity to have an additional information on the history of the pressure both at $x=0$ (gas supply) and at $x=2 l$ (gas measurement). Let

$$
\tilde{P}(0, t)=\tilde{P}_{0}(0)=\tilde{P}_{0}, \quad \tilde{P}(2 l, t)=\tilde{P}_{2 l} .
$$

Let us write the functional (30) for the pressure $P(x, t)$,

$$
R_{P}(\lambda)=\int_{0}^{T}[P(2 l, t)-\tilde{P}(2 l, t)]^{2} d t
$$

where at the point $x=2 l$ from (34) we have

$$
P(2 l, t)=P_{l+0}-\frac{2 a_{2}}{F_{2}} Q_{l+0} 2 l .
$$

Taking into account that there is no dependence from $t$ either in (35) or (37), the functional (36) takes the form

$$
R_{P}(\lambda)=\left(P(2 l, t)-\tilde{P}_{2 l}\right)^{2} T=\left(P_{l+0}-\frac{2 a_{2}(\lambda)}{F_{2}} Q_{l+0} l-\tilde{P}_{2 l}\right)^{2} T .
$$

Now, similarly as in (31), we have the gradient of the functional in the form

$$
a_{2}(\lambda)=\frac{F_{2}}{4 l Q(l+0)}\left(P_{l+0}-\tilde{P}_{2 l}\right) .
$$

Taking into account (39), in (17), for $\lambda$, we have

$$
\lambda=-\frac{2 D_{2} g}{\omega_{0} \omega_{2}}+\frac{F_{2} D_{2}}{2 \omega_{2} l Q(l+0)}\left(P_{l+0}-\tilde{P}_{2 l}\right) .
$$


Now we will show the above on the numerical example. Let for $0 \leq x<l, l=$ $1485 \mathrm{~m}, c_{1}=331 \mathrm{~m} / \mathrm{c}, \rho_{1}=0,717: 3 / \mathrm{m}^{3}, F_{1}=0.006, \lambda_{1}=0.01, D_{1}=$ $0.0876, g=9.81, \omega_{1}=48.6390$, and for $l \leq x<2 l$,

$c=850, D_{2}=0.073, F_{2}=0.0042, \omega_{2}=0.0546, \lambda_{2}=0.23, \bar{Q}=100, \bar{P}=100$.

Performing the corresponding calculations using the values of the latter parameters, we have:

$$
\begin{gathered}
P_{0}-\frac{2 a_{1}}{F_{1}} Q_{0} l=-2.466 \cdot 10^{3}, \\
P_{l+0}=P_{l-0}+P_{l-0}^{2} \cdot \bar{P}=1.3494 \cdot 10^{15}, \\
P_{l-0}=P_{0}-2 \frac{a_{1}}{F_{1}} Q_{0} l=-3.6734 \cdot 10^{6}, \\
Q_{l+0}=Q_{0}-Q_{0}^{2} \bar{Q}=-2495, \\
Q_{0}=0.001, \quad \bar{P}=0.00135, \quad \tilde{P}(2 l, t)=\tilde{P}_{2 l}=1.34 \cdot 10^{15} .
\end{gathered}
$$

Then, for the value $\tilde{P}_{2 l}=1.34 \cdot 10^{15}$ from (40), we can find $\lambda^{*}$ in the form $\lambda^{*}=2.2999 \cdot 10^{-1}$ which coincides with $\lambda_{2}$ from (41) with an accuracy of $10^{-3}$. Thus, the stated problems can be extended to the more difficult problem (1)-(3).

\section{References}

[1] V.I. Shurov, Technology and Techniques of Oil Recovery. Nedra, Moscow, 1983.

[2] E. Camponogara, A. Plucenio, A.F. Teixeira, and S.R.V. Campos, An Automation System for Gas-Lifted Oil Wells: Model Identification Control and Optimization. J. Petroleum Sci. and Engineering 70 (2010), 157-167.

[3] F.A. Aliev, N.A. Ismailov, and N.S. Mukhtarova, Algorithm to Determine the Optimal Solution of a Boundary Control Problem. - Automation and Remote Control 76 (2015), No. 4, 627-633.

[4] F.A. Aliev, M.Kh. Ilyasov, and M.A. Dzamalbekov, Modeling of Work of Gas-Lift Wells. - Rep. of NAS of Azerbaijan 4 (2008), 30-41.

[5] F.A. Aliev, M.Kh. Ilyasov, and N.B. Nuriev, The Problems of Modeling and Optimal Stabilization of Gas Lift Process. - Appl. Mechanics (2010), No. 6, 115-122.

[6] F.A. Aliev, M.M. Mutallimov, I.M. Askerov, and I.S. Raguimov, Asymptotic Method of Solution for a Problem of Construction of Optimal Gas-lift Process Modes. Mathematical Problems in Engineering (2010), Article ID 191053, 10 pages. 
[7] F.A. Aliev, N.A. Aliev, K.G. Hasanov, A.K. Turarov, A.P. Guliev, and G.V.Isaeva, Numerical-Analytical Method for Solving of the First Order Partial Quasi-Linear Equations, TWMS J. - Pure Appl. Math. 6 (2015), No. 2, 158-164.

[8] M. Ashraf, S. Asghar, and M.A. Hossain, The Computational Study of the Effects of Magnetic Field and Free Stream Velocity Oscillation on Boundary Layer Flow Past a Magnetized Vertical Plate. - Appl. Comput. Math. 13 (2014), No. 2, 175-193.

[9] A.Kh. Mizadjanzadeh, I.M. Ametov, A.M. Khasaev, and V.I. Gusev, Technology and Technique of Oil Extractions. Nedra, Moscow, 1986.

[10] F.A. Aliev, M.M. Mutallimov, N.A. Ismailov, and M.F. Racabov, An Algorithm for Constructing Optimal Controllers for Gaslift Operation. - Automation and Remote Control 73 (2012), No. 8, 12791289.

[11] F.A. Aliev, M.A. Djamalbekov, and M.Kh. Ilyasov, Mathematical Simulation and Control of Gas-Lift. - J. Computer and Systems Sciences International 50 (2011), No. 1, 805-814.

[12] F.A. Aliev and N.A. Ismailov, Problems of Optimization with the Periodic Boundary Condition and Boundary Control in Gas Lift Wells. - Nonlinear Vibrations (2014), No. 2, 216-227.

[13] A.P. Guliev, M.Kh. Ilyasov, N.A. Aliev, and F.A. Aliev, The Algorithm for Solving the Problem of Determining the Motion of Space Process. - Proc. IAM 2 (2013), No. 1, 91-97.

[14] A.O. Gelfand, Calculation of Finite Differences. Nauka, Moscow, 1967.

[15] N.A. Aliev, F.A. Aliev, A.P. Guliev, and M.Kh. Ilyasov, Method of Series in the Solution of One Boundary Problem for the System of Hyperbolic Type Equations, Arising in the Oil Production. - Proc. IAM 2 (2013), No. 2, 113-136.

[16] S. Alkan and A. Secer, Solution of Nonlinear Fractional Boundary Value Problems with Nonhomogeneous Boundary Conditions. - Appl. Comput. Math. 14 (2015), No. 3, 284-295.

[17] J. Rashidinia, M. Khazaei, and H. Nikmarvani, Spline Collocation Method for Solution of Higher Order Linear Boundary Value Problems, TWMS J. - Pure Appl. Math. 6 (2015), No. 1, 38-47.

[18] L.K. Vashisht, Brief Paper. Banach Frames Generated by Compact Operators Associated with a Boundary Value Problem, TWMS J. - Pure Appl. Math. 6 (2015), No. 2, 254-258.

[19] F.A. Aliev and N.A. Ismailov, Inverse Problem to Determine the Hydraulic Resistance Coefficient in the Gas Lift Process. - Appl. Comput. Math. 12 (2013), No. $3,306-313$.

[20] F.A. Aliev, N.A. Ismailov, and A.A. Namazov, Asymptotic Method for Finding the Coefficient of Hydraulic Resistance in Lifting of Fluid on Tubing. - Inverse and Ill Posed Problems 23 (2015), No. 5, 511-518. 
[21] G.D. Birkhoff, On the Asymptotic Character of the Solutions of Certain Linear Differential Equations Containing a Parameter. - Trans. Am. Math Soc. 9 (1908), 219-231; Boundary Value and Expansion Problems of Ordinary Linear Differential Equations. $A M S 9$ (1908), No. 4.

[22] A. Erdeyi, Asymptotic Decompositions. Moscow, 1962.

[23] S. Tiwari and M. Kumar, An Initial Value Technique to Solve Two-Point NonLinear Singularly Perturbed Boundary Value Problems. - Appl. Comput. Math. 14 (2015), No. 2, 150-157.

[24] Hector Vazquez-Leal, The Enhanced Power Series Method to Find Exact or Approximate Solutions of Nonlinear Differential Equations. - Appl. Comput. Math. 14 (2015), No. 2, 168-179.

[25] M.A. Lavrentyev and V.V. Shabat, Methods of Theory of Functions of Complex Variables. Nauka, Moscow, 1973.

[26] Z.O. Melnik, Solution of a Mixed Problem for Hyperbolic Equations with Discontinuous Coefficients. - Differ. Eqs. 2 (1966), No. 4, 560-570.

[27] M. Mohammadi, R. Mokhtari, and H. Panahipour, Solving Two Parabolic Inverse Problems with a Nonlocal Boundary Condition in the Reproducing Kernel Space. - Appl. Comput. Math. 13 (2014), No. 1, 91-106. 